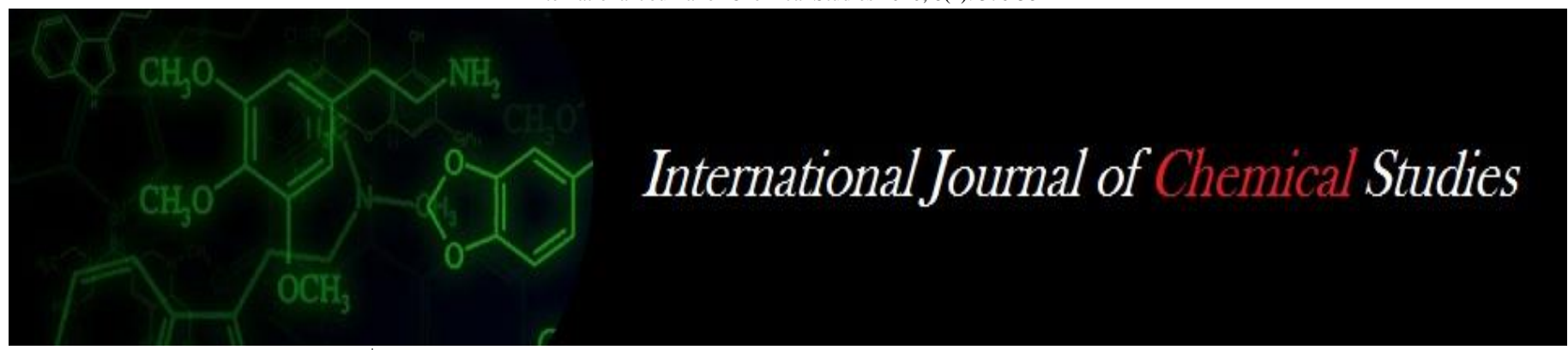

P-ISSN: 2349-8528

E-ISSN: 2321-4902

IJCS 2020; 8(1): 376-382

(C) 2020 IJCS

Received: 22-11-2019

Accepted: 24-12-2019

Rajendra Hegde ${ }^{1}$

Principal Scientist \&Head,

ICAR-National Bureau of Soil

Survey and Land Use Planning,

Regional Centre, Bengaluru,

Karnataka, India

SP Chaitra

Senior Research Fellow ICAR-

National Bureau of Soil Survey and Land Use Planning,

Regional Centre, Bengaluru,

Karnataka, India

\section{TN Somashekar}

Senior Research Fellow ICAR-

National Bureau of Soil Survey

and Land Use Planning,

Regional Centre, Bengaluru,

Karnataka, India

\section{BA Dhanorkar}

Chief Technical Officer ICAR-

National Bureau of Soil Survey

and Land Use Planning,

Regional Centre, Bengaluru,

Karnataka, India

\section{KV Niranjana}

Chief Technical Officer ICARNational Bureau of Soil Survey and Land Use Planning,

Regional Centre, Bengaluru,

Karnataka, India

\section{P Chandran}

Director (Acting) ICAR-National Bureau of Soil Survey and Land Use Planning, Nagpur,

Maharashtra, India
Corresponding Author: SP Chaitra

Senior Research Fellow ICARNational Bureau of Soil Survey and Land Use Planning, Regional Centre, Bengaluru, Karnataka, India

\section{Characterisation and classification of soils of parts of Alur, Khairat, Matki and Padasavli subwatersheds, Aland Taluk, Kalaburagi district, Karnataka}

\author{
Rajendra Hegde, SP Chaitra, TN Somashekar, BA Dhanorkar, KV \\ Niranjana and P Chandran
}

DOI: https://doi.org/10.22271/chemi.2020.v8.i1e.8275

\begin{abstract}
Land Resource Inventory was undertaken to characterize and classify the soils of parts of Alur, Khairat, Matki and Padasavli subwatersheds covering an area of 5509 ha in Aland Taluk, Kalaburagi district of Karnataka. Two hundred fifteen soil profiles were studied for the morphological, physical and chemical properties and 10 soil series were identified. Representative pedons covering all the soil series were selected and their properties and classification is discussed in this paper. The soils were very shallow to very deep in depth, very dark grayish brown to dark yellowish brown and very dark gray in black soils and dark reddish-brown colour in red soils. They are predominantly sub- angular blocky in structure, clay in texture, neutral to alkaline in soil reaction and organic carbon content is high. Soils studied were classified up to series level according to Soil Taxonomy using morphological, physical and chemical properties. Inceptisols cover a maximum area of 2299 ha (42\%) followed by Entisols 1845 ha (34\%) and Vertisols 1214 ha $(22 \%)$ in these subwatersheds.
\end{abstract}

Keywords: Land resource inventory, soil series, soil characterisation and soil classification

\section{Introduction}

Land is a scarce resource and basic unit for any material production. It can support the needs of the growing population, provided they use the land in a rational and judicious manner. But what is happening in many areas of the state is a cause for concern to everyone involved in the management of land resources at the grassroots level. Hence, there is an urgent need to generate detailed site-specific farm level database on various land resources for all the villages/watersheds in a time bound manner that would help to protect the valuable soil and land resources and also to stabilize the farm production. Therefore, the land resource inventory required for farm level planning is the one which investigates all the parameters which are critical for productivity viz., soils, site characteristics like slope, erosion, gravelliness and stoniness, climate, water, topography, geology and various schemes and developmental works of the government etc. No information is available on the characterization, classification and genesis of soils of Kalaburagi district. Hence, the present study was taken up to characterize, classify and evaluate the soils of parts of Khairat, Matki, Alur and Padasavli subwatersheds in Aland taluk of Kalaburagi district.

\section{Materials and Methods}

The study area forms a part of Khairat, Matki, Alur and Padasavli subwatersheds in the northeastern part of Karnataka in Aland Taluk, Kalaburagi District, Karnataka State (Fig 1.). It lies between $76^{\circ} 23^{\prime} 14^{\prime \prime}$ ' and $76^{\circ} 31^{\prime}$ '24' 'East longitudes and $17^{\circ} 31^{\prime} 22^{\prime \prime}$ ' and $17^{\circ} 40^{\prime} 0.05^{\prime}$ ', North latitudes with an elevation of $600 \mathrm{~m}$ above MSL and covers an area of 5509 ha. It is about $15 \mathrm{~km}$ from Aland town and the subwatersheds comprise 9 microwatersheds namely, Nirgudi-1, Nirgudi-2, Nirgudi West, Chincholi Khurd-1, Chincholi Khurd-2, Padasavli-1, Padasavli-2, Padasavli-3 and Matki-3 covering parts of Nirgudi, Matki and Padasavli villages. The study area falls under semiarid tract of the state and is categorized as drought prone with average annual rainfall of $785 \mathrm{~mm}$. 


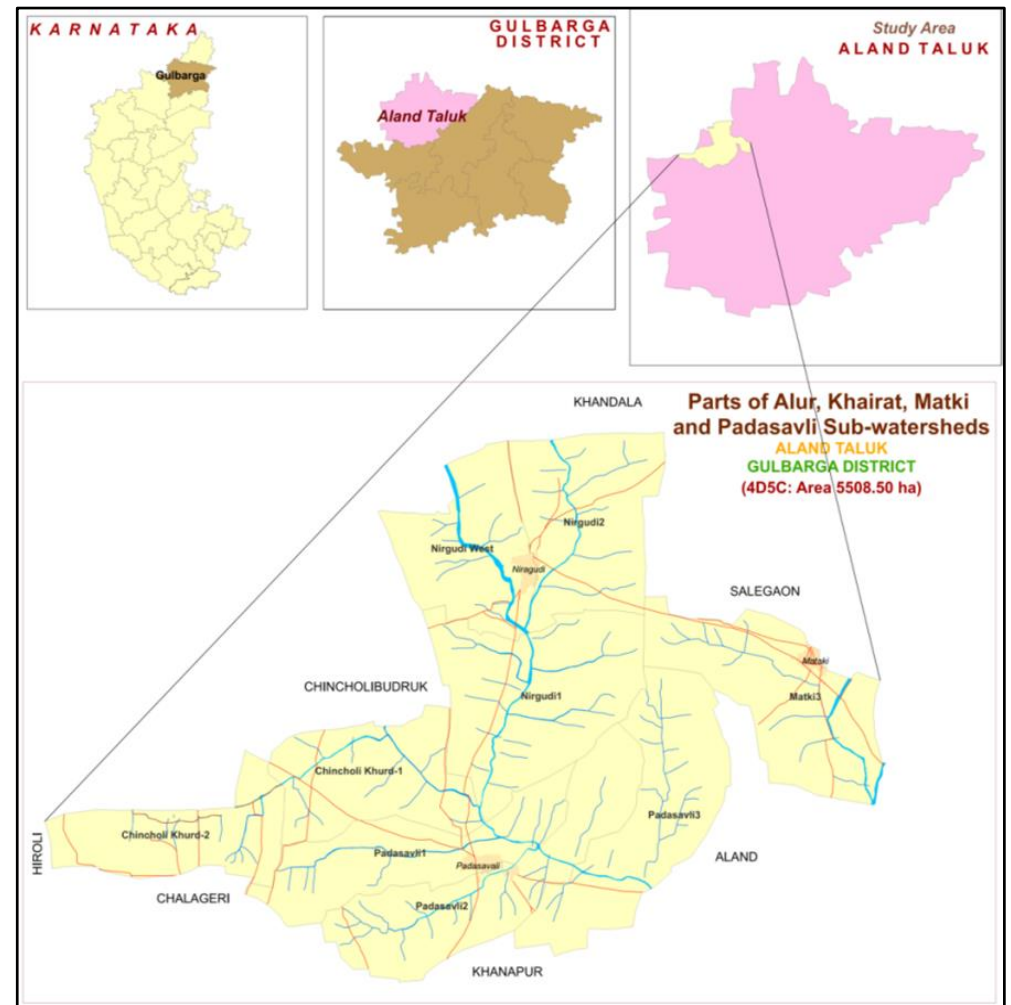

Fig 1: Location map of parts of Khairat, Matki, Alur and Padasavli subwatersheds

The land resource inventory was conducted using village cadastral maps and IRS satellite imagery on 1:7920 scale (Fig. 2). Physiographycally the area has been identified as basalt and laterite landscape. The false colour composites of IRS imagery were interpreted for physiography and the physiographic delineations were used as base for mapping soils. The soils were studied in several transects by locating soil profiles at closely spaced intervals to take care of any change in the land features like break in slope, erosion, gravel, stones etc.
In the selected sites, profiles (vertical cut showing the soil layers from surface to the rock) were opened up to $200 \mathrm{~cm}$ or to the depth limited by rock or hard substratum and studied in detail for all their morphological and physical characteristics. The soil and site characteristics were recorded for all profile sites on a standard proforma as per the guidelines given in USDA Soil Survey Manual (Soil Survey Staff, 2012). In all, about 215 profiles were studied.

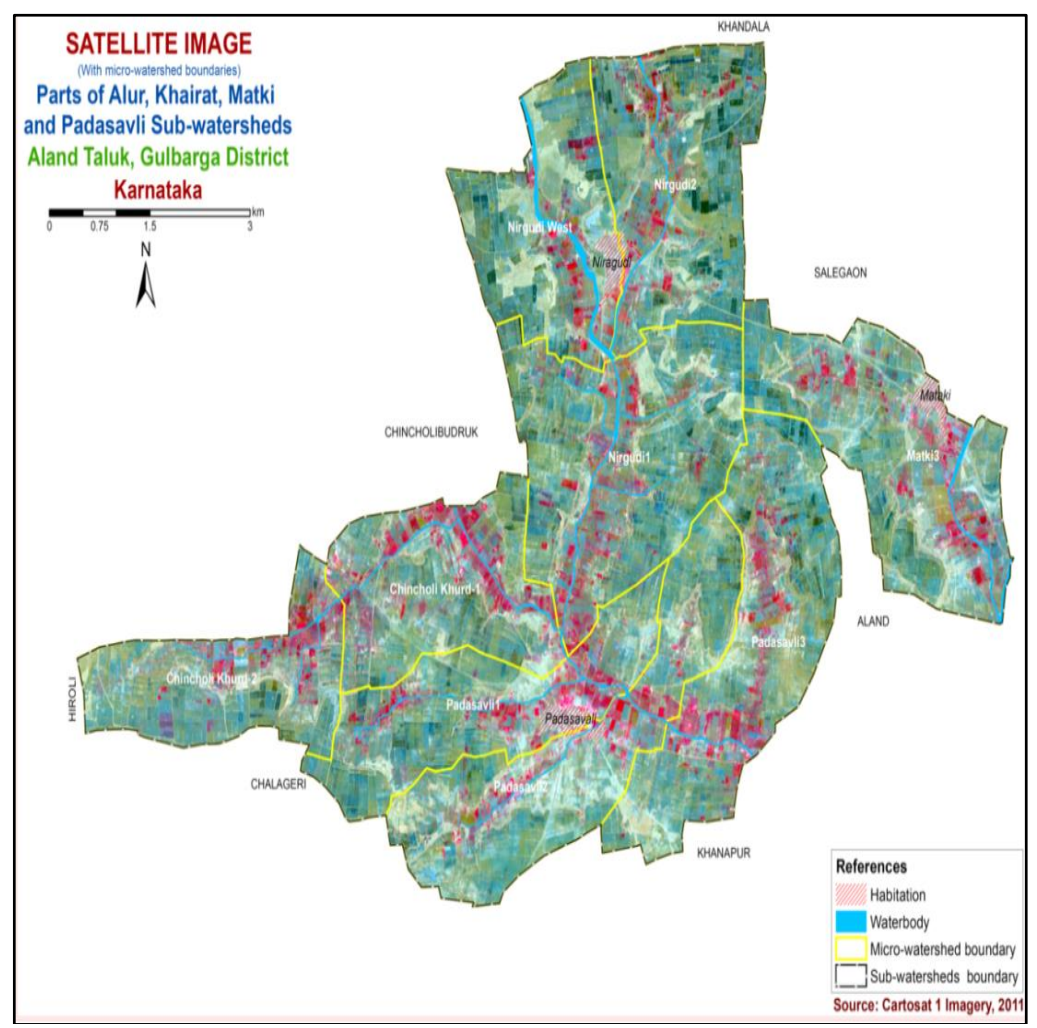

Fig 2: Satellite Image of parts of Khairat, Matki, Alur and Padasavli subwatersheds 
Based on the soil characteristics, the soils were grouped into different soil series. Soil series is the most homogeneous unit having similar horizons and properties and behaves similarly for a given level of management. Soil depth, texture, colour, kind of horizon and horizon sequence, amount and nature of gravel present, calcareousness, nature of substratum etc, were used as the major differentiating characteristics for identifying soil series occurring in the area. The differentiating characteristics used for identifying the soil series are given in Table 1. Ten soil series were identified and mapped as phases of soil series (management units) and a soil map was prepared with phases of soil series as mapping units. Random checks were made all over the area outside the transects to confirm and validate the soil map unit boundaries. The soil map shows the geographic distribution and extent, characteristics, classification, behavior and use potentials of the soils in the Subwatershed. The soil map prepared with phases of soil series was categorically and cartographically generalised and a soil series was prepared (Fig.3). Soil samples for each series were collected from representative master profiles for laboratory characterization by following the methods outlined in the Laboratory Manual (Sarma et al. 1987) ${ }^{[6]}$.

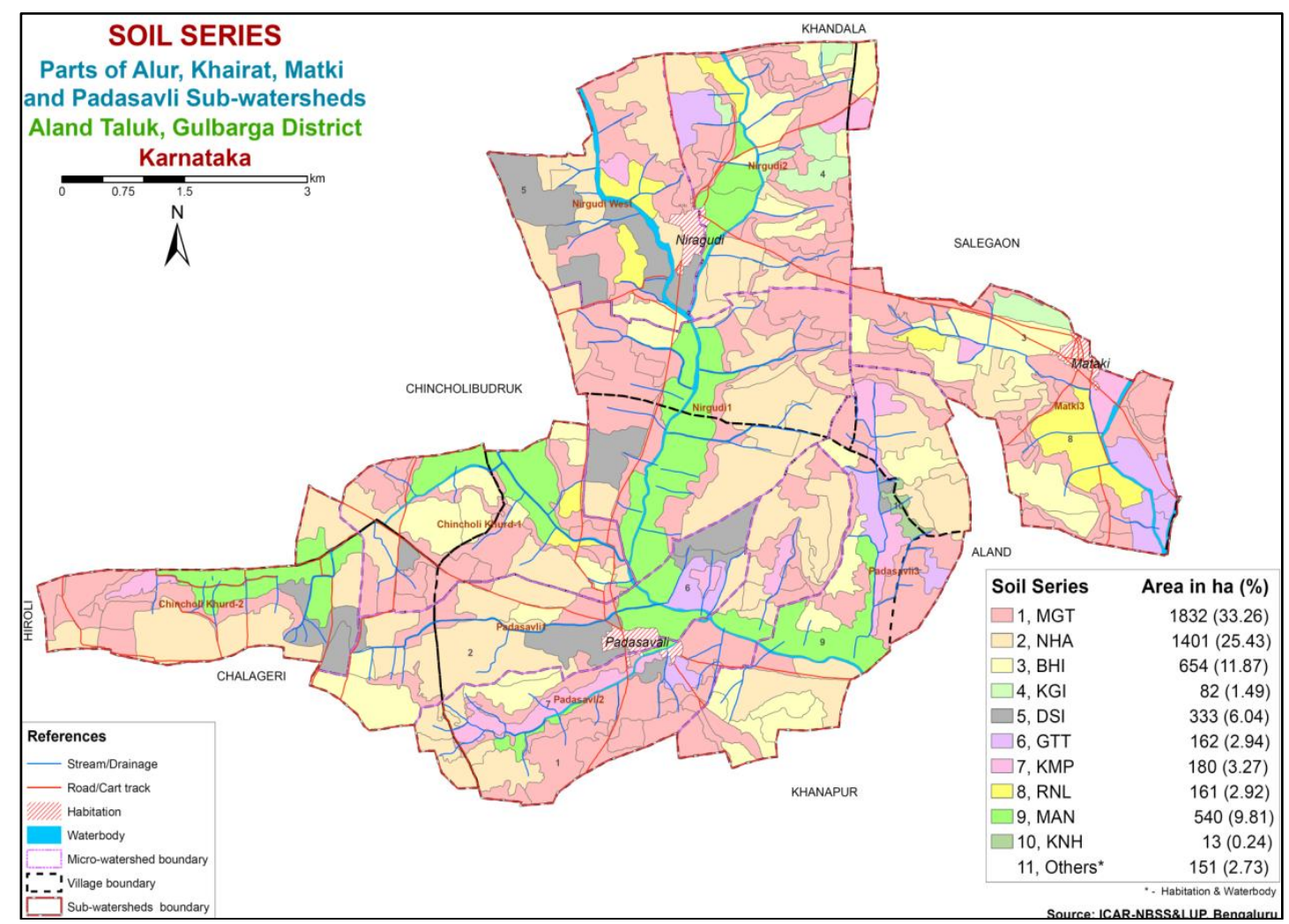

Fig 3: Soil Series map of parts of Khairat, Matki, Alur and Padasavli Subwatersheds

Table 1: Differentiating Characteristics used for Identifying soil series (Characteristics are of series control section)

\begin{tabular}{|c|c|c|c|c|c|c|c|}
\hline SI No. & Series & Depth (cm) & Colour (moist) & Texture & Gravel (\%) & Horizon Sequen & Effervescence \\
\hline \multicolumn{8}{|c|}{ Soils of Basalt landscape } \\
\hline 1 & Margutti (MGT) & $<25$ & 10YR3/3,4/3,5/4 7.5YR4/3 & $\mathrm{gc}$ & $15-35$ & Ap-Ac & - \\
\hline 2 & Novinihala (NHA) & $25-50$ & 10YR3/2,3/1,4/2 7.5YR3/4 & gcl & $15-35$ & Ap-Bw & - \\
\hline 3 & Bhimanahalli (BHI) & $25-50$ & 10YR3/2,3/3,3/1 7.5YR3/2,4/2 & gc & $15-35$ & Ap-Bw-CB & - \\
\hline 4 & Kalamundargi (KGI) & $25-50$ & 10YR4/3,4/2 7.5YR3/3,3/4,4/3 & gcl & $35-60$ & Ap-Bw & - \\
\hline 5 & Dinsi (DSI) & $50-75$ & 10YR3/2,3/3,4/3, 3/2 & $\mathrm{c}$ & $<15$ & Ap-Bss & - \\
\hline 6 & Gutti (GTT) & $50-75$ & 10YR3/2,3/ 7.5YR3/3,4/3 & $\mathrm{gc}$ & $15-35$ & $\mathrm{Ap}-\mathrm{Bw}-\mathrm{Bc}$ & - \\
\hline 7 & Kamalapur (KMP) & $75-100$ & $10 \mathrm{YR} 3 / 2,3 / 1$ & $\mathrm{c}$ & $<15$ & Ap-AB-Bss & - \\
\hline 8 & Rajanala (RNL) & $100-150$ & $10 \mathrm{YR} 3 / 2,3 / 1,4 / 2,4 / 3$ & $\mathrm{c}$ & $<15$ & Ap-A1-Bss & es \\
\hline 9 & Mahagaon (MAN) & $>150$ & \begin{tabular}{|l|l}
$10 \mathrm{YR} 3 / 2,3 / 1$ \\
\end{tabular} & $\mathrm{c}$ & $<15$ & Ap-A1-Bss & es \\
\hline \multicolumn{8}{|c|}{ Soils of Laterite landscape } \\
\hline 10 & Kinhi $(\mathrm{KNH})$ & $<25$ & 2.5YR3/3 5YR4/6 & $\mathrm{gcl}$ & $35-60$ & Ap & - \\
\hline
\end{tabular}

\section{Results and Discussion}

\section{Morphological Characteristics of Soils}

Of the, 10 soil series, 9 soil series were identified under basalt landscape and one soil series in laterite landscape. The brief morphological characteristics of the soil series are presented in Table 2.

The soils are very shallow to very deep have, very dark grayish brown (10 YR 3/2) to dark brown (10 YR 3/3, 7.5 YR3/3) in surface horizons and dark yellowish brown (10 YR $3 / 4)$ to very dark gray (10 YR 3/1) in subsurface horizons of all the soil series, except Kinhi $(\mathrm{KNH})$ series that belongs to the lateritic landscape that is dark reddish brown (2.5 YR 3/3) in colour. There was not much variation in the soil colour with depth in all the series. The dominant colour in the black soil pedons vary from very dark grayish brown to very dark gray due to the clay-humus complex in the presence of lime. The dark matrix colour was due to the presence of high organic matter content in the surface horizons (Tripathi et al. 2006) ${ }^{[10]}$. In the kinhi soil series, hue was (2.5 YR). The soil colour appears to be the function of chemical and 
mineralogical composition as well as textural make up of soils and conditioned by topographic position and moisture regime (Walia and Rao 1997) ${ }^{[11]}$.

The texture of the Margutti (MGT), Bhimanahalli (BHI), Dinsi (DSI), Gutti (GTT), Kamalapur (KMP), Rajnala (RNL) and Mahagaon (MAN) soil series were found to be clayey, whereas, Novinihala (NHA), Kakamundargi (KGI) and Kinhi $(\mathrm{KNH})$ were found to be loamy. This textural variation might be due to differences in composition of parent material, topography, in-situ weathering and translocation of clay by eluviations and age of soils (Geetha Sireesha and Naidu 2013) [2].

Table 2: Morphological Characteristics of soils of Parts of Alur, Khairat, Matki and Padasavli subwatersheds

\begin{tabular}{|c|c|c|c|c|c|c|c|c|c|c|c|c|c|c|}
\hline \multirow{2}{*}{ Horizon } & \multirow{2}{*}{$\begin{array}{l}\text { Depth } \\
(\mathbf{c m})\end{array}$} & \multirow{2}{*}{$\begin{array}{l}\text { Diagnostic } \\
\text { horizon }\end{array}$} & \multirow{2}{*}{$\begin{array}{l}\text { Colour } \\
\text { (moist) }\end{array}$} & \multirow{2}{*}{$\begin{array}{c}\text { Texture }^{1} \\
\text { Class } \\
\text { (USDA) }\end{array}$} & \multirow{2}{*}{$\begin{array}{c}\text { Coarse }^{2} \text { fragments } \\
\text { w/w }(\%)\end{array}$} & \multicolumn{3}{|c|}{ Structure $^{3}$} & \multicolumn{4}{|c|}{ Consistence $^{4}$} & \multicolumn{2}{|c|}{ Boundary } \\
\hline & & & & & & Grade & Size & Type & Dry & Moist & Stickyness & splasticity & D & $\mathbf{T}$ \\
\hline \multicolumn{15}{|c|}{ Soils of Basalt landscape } \\
\hline \multicolumn{15}{|c|}{ Margutti (MGT) series } \\
\hline $\mathrm{Ap}$ & $0-15$ & Ochric & $10 \mathrm{YR} 3 / 2$ & $\mathrm{c}$ & 20 & 2 & $\mathrm{~m}$ & sbk & sh & $\mathrm{fr}$ & ss & $\mathrm{sp}$ & $\mathrm{c}$ & $\mathrm{s}$ \\
\hline $\mathrm{AC}$ & $15-25$ & & $10 Y R 4 / 4$ & $\mathrm{c}$ & 80 & 2 & $\mathrm{~m}$ & sbk & sh & $\mathrm{fr}$ & Ss & $\mathrm{sp}$ & $\mathrm{c}$ & $\mathrm{s}$ \\
\hline \multicolumn{15}{|c|}{ Novinihala (NHA) series } \\
\hline Ap & $0-15$ & Ochric & $10 Y R 3 / 3$ & scl & 10 & 2 & $\mathrm{~m}$ & sbk & sh & $\mathrm{fr}$ & ss & $\mathrm{sp}$ & $\mathrm{c}$ & $\mathrm{s}$ \\
\hline $\mathrm{Bw}$ & $15-40$ & Cambic & 10YR3/3 & $\mathrm{cl}$ & 20 & 2 & $\mathrm{~m}$ & sbk & sh & fr & Ss & $\mathrm{sp}$ & $\mathrm{c}$ & $\mathrm{s}$ \\
\hline \multicolumn{15}{|c|}{ Bhimanahalli (BHI) series } \\
\hline Ap & $0-6$ & Ochric & $10 Y R 4 / 3$ & $\mathrm{c}$ & - & 2 & $\mathrm{~m}$ & sbk & sh & $\mathrm{fr}$ & ss & $\mathrm{sp}$ & $\mathrm{c}$ & $\mathrm{s}$ \\
\hline $\mathrm{Bw} 1$ & $6-25$ & Cambic & $10 \mathrm{YR} 4 / 4$ & $\mathrm{c}$ & - & 2 & $\mathrm{~m}$ & sbk & sh & $\mathrm{fr}$ & ss & $\mathrm{sp}$ & $\mathrm{c}$ & $\mathrm{s}$ \\
\hline $\mathrm{CB}$ & $25-45$ & & $10 \mathrm{YR} 4 / 4$ & sl & - & 2 & $\mathrm{~m}$ & sbk & sh & $\mathrm{fr}$ & ss & $\mathrm{sp}$ & $\mathrm{c}$ & $\mathrm{s}$ \\
\hline \multicolumn{15}{|c|}{ Kalamundargi (KGI) series } \\
\hline Ap & $0-10$ & Ochric & 10YR3/2 & $\mathrm{cl}$ & 20 & 2 & $\mathrm{~m}$ & sbk & sh & $\mathrm{fr}$ & ss & $\mathrm{sp}$ & $\mathrm{c}$ & $\mathrm{s}$ \\
\hline $\mathrm{Bw}$ & $10-28$ & Cambic & 10YR3/4 & $\mathrm{cl}$ & 50 & 2 & $\mathrm{~m}$ & sbk & sh & fr & ss & $\mathrm{sp}$ & $\mathrm{c}$ & $\mathrm{s}$ \\
\hline \multicolumn{15}{|c|}{ Dinsi (DSI) series } \\
\hline $\mathrm{Ap}$ & $0-10$ & Ochric & $10 \mathrm{YR} 3 / 2$ & $\mathrm{c}$ & 05 & 2 & $\mathrm{~m}$ & sbk & sh & fr & Ss & $\mathrm{sp}$ & $\mathrm{c}$ & $\mathrm{s}$ \\
\hline Bss1 & $10-30$ & Vertic & 10YR3/1 & $\mathrm{c}$ & - & 2 & $\mathrm{~m}$ & abk & sh & fi & vs & $\mathrm{vp}$ & $\mathrm{c}$ & $\mathrm{s}$ \\
\hline Bss2 & $30-50$ & Vertic & 10YR3/1 & c & - & 2 & $\mathrm{~m}$ & abk & sh & fi & vs & $\mathrm{vp}$ & $\mathrm{c}$ & $\mathrm{s}$ \\
\hline Bss3 & $50-75$ & Vertic & 10YR3/1 & $\mathrm{c}$ & 05 & 2 & $\mathrm{~m}$ & abk & sh & fi & vs & vp & $\mathrm{c}$ & $\mathrm{s}$ \\
\hline \multicolumn{15}{|c|}{ Gutti (GTT) series } \\
\hline Ap & $0-15$ & Ochric & $7.5 Y R 3 / 3$ & $\mathrm{c}$ & 05 & 2 & $\mathrm{~m}$ & sbk & sh & $\mathrm{fr}$ & ss & sp & $\mathrm{c}$ & $\mathrm{s}$ \\
\hline Bw1 & $15-40$ & Cambic & $7.5 Y R 3 / 4$ & $\mathrm{c}$ & 20 & 2 & $\mathrm{~m}$ & sbk & sh & $\mathrm{fr}$ & Ss & sp & $\mathrm{c}$ & $\mathrm{s}$ \\
\hline $\mathrm{BC}$ & $40-75$ & & $7.5 \mathrm{YR} 3 / 4$ & c & 50 & 2 & $\mathrm{~m}$ & sbk & sh & $\mathrm{fr}$ & Ss & sp & $\mathrm{c}$ & $\mathrm{s}$ \\
\hline \multicolumn{15}{|c|}{ Kamalapur (KMP) series } \\
\hline Ap & $0-10$ & Ochric & 10YR3/2 & $\mathrm{c}$ & - & 2 & $\mathrm{~m}$ & sbk & sh & $\mathrm{fr}$ & SS & $\mathrm{sp}$ & $\mathrm{c}$ & $\mathrm{s}$ \\
\hline $\mathrm{AB}$ & $10-30$ & Ochric & $10 \mathrm{YR} 3 / 2$ & $\mathrm{c}$ & - & 2 & $\mathrm{~m}$ & sbk & sh & $\mathrm{fr}$ & ss & $\mathrm{sp}$ & $\mathrm{c}$ & $\mathrm{s}$ \\
\hline Bss1 & $30-43$ & Vertic & $10 \mathrm{YR} 3 / 2$ & $\mathrm{c}$ & - & 2 & $\mathrm{~m}$ & abk & sh & fi & $\mathrm{ms}$ & $\mathrm{sp}$ & $\mathrm{c}$ & $\mathrm{s}$ \\
\hline Bss2 & $43-60$ & Vertic & $10 \mathrm{YR} 3 / 2$ & $\mathrm{c}$ & - & 2 & $\mathrm{~m}$ & abk & sh & fi & $\mathrm{ms}$ & sp & $\mathrm{c}$ & $\mathrm{s}$ \\
\hline $\mathrm{BC}$ & $60-85$ & & $10 Y R 4 / 4$ & $\mathrm{c}$ & - & 2 & $\mathrm{~m}$ & sbk & sh & $\mathrm{fr}$ & SS & $\mathrm{sp}$ & $\mathrm{c}$ & $\mathrm{s}$ \\
\hline \multicolumn{15}{|c|}{ Rajanala (RNL) series } \\
\hline Ap & $0-18$ & Ochric & 10YR3/2 & $\mathrm{c}$ & 05 & 2 & $\mathrm{~m}$ & sbk & sh & $\mathrm{fr}$ & ss & $\mathrm{sp}$ & $\mathrm{c}$ & $\mathrm{s}$ \\
\hline A1 & $18-40$ & Ochric & $10 \mathrm{YR} 3 / 2$ & $\mathrm{c}$ & 05 & 2 & $\mathrm{~m}$ & sbk & sh & $\mathrm{fr}$ & ss & $\mathrm{sp}$ & $\mathrm{c}$ & $\mathrm{s}$ \\
\hline Bss1 & $40-60$ & Vertic & $10 \mathrm{YR} 3 / 2$ & $\mathrm{c}$ & - & 2 & $\mathrm{~m}$ & abk & sh & fi & vs & $\mathrm{vp}$ & $\mathrm{c}$ & $\mathrm{s}$ \\
\hline Bss2 & $60-105$ & Vertic & $10 Y R 3 / 2$ & $\mathrm{c}$ & - & 2 & $\mathrm{~m}$ & abk & sh & fi & vs & $\mathrm{vp}$ & $\mathrm{c}$ & $\mathrm{s}$ \\
\hline Bss3 & $105-150$ & Vertic & $10 \mathrm{YR} 3 / 2$ & $\mathrm{c}$ & - & 2 & $\mathrm{~m}$ & abk & \begin{tabular}{|l|}
$\mathrm{sh}$ \\
\end{tabular} & fi & vs & $\mathrm{vp}$ & $\mathrm{c}$ & $\mathrm{s}$ \\
\hline \multicolumn{15}{|c|}{ Mahagaon (MAN) series } \\
\hline $\mathrm{Ap}$ & $0-20$ & Ochric & $10 Y R 3 / 2$ & $\mathrm{c}$ & - & 2 & $\mathrm{~m}$ & sbk & sh & fr & ss & $\mathrm{sp}$ & $\mathrm{c}$ & $\mathrm{s}$ \\
\hline A1 & $20-45$ & Ochric & $10 \mathrm{YR} 3 / 2$ & $\mathrm{c}$ & - & 2 & $\mathrm{~m}$ & sbk & sh & fr & ss & $\mathrm{sp}$ & $\mathrm{c}$ & $\mathrm{s}$ \\
\hline Bss1 & $45-75$ & Vertic & $10 Y R 3 / 2$ & $\mathrm{c}$ & - & 2 & $\mathrm{~m}$ & abk & sh & $\mathrm{fi}$ & vs & $\mathrm{vp}$ & $\mathrm{c}$ & $\mathrm{s}$ \\
\hline Bss2 & $75-110$ & Vertic & $10 \mathrm{YR} 3 / 2$ & $\mathrm{c}$ & - & 2 & $\mathrm{~m}$ & abk & sh & fi & vs & $\mathrm{vp}$ & $\mathrm{c}$ & $\mathrm{s}$ \\
\hline Bss3 & $110-140$ & Vertic & $10 Y R 4 / 3$ & $\mathrm{c}$ & - & 2 & $\mathrm{~m}$ & abk & sh & fi & vs & $\mathrm{vp}$ & $\mathrm{c}$ & $\mathrm{s}$ \\
\hline & & & & & s of Laterite landsca & & & & & & & & & \\
\hline & & & & & Kinhi (KNH) series & & & & & & & & & \\
\hline Ap & $0-18$ & Ochric & $2.5 \mathrm{YR} 3 / 3$ & $\mathrm{cl}$ & 55 & 2 & $\mathrm{~m}$ & sbk & sh & fr & ss & $\mathrm{sp}$ & $\mathrm{c}$ & $\mathrm{s}$ \\
\hline
\end{tabular}

Note: 1 . Texture: sl-sandy loam, scl-sandy clay loam, cl-clay loam, sc-sandy clay, c -clay.

2. Size: fg- fine gravel $(<2 \mathrm{~cm})$, cg-coarse gravel $(2-7.5 \mathrm{~cm})$, cb-cobbles $(7.5-25 \mathrm{~cm})$, st-stones $(25-60 \mathrm{~cm})$, b-boulders $(>60 \mathrm{~cm})$.

3. Grade: 0-structureless, 1-weak, 2- moderate, 3-strong; Size: vf-very fine, f-fine, m-medium, c-coarse, vc-very coarse Type: gr-granular, crcrumb, clr-columnar, pr-prismatic, pl-platy, abk-angular blocky, sbk-subangular blocky, sg-single grain, m-massive, c-cloddy.

4. Dry: 1-loose, s-soft, sh-slightly hard, h-hard, vh-very hard, eh-extremely hard, Moist: 1-loose, vfr-very friable, fr-friable, fi-firm, vfi-very firm, efi-extremely firm, Stickyness: so-non-sticky, ss-slightly sticky, ms-moderately sticky, vs-very sticky, Plasticity: po-non-plastic, sp- slightly plastic, mp-moderately plastic, vp-very plastic.

5. D-Distinctness: a-abrupt, c-clear, g-gradual, d-diffuse, T-topography: s-smooth, w-wavy, i -irregular, b-broken

The structure indicates the mode of arrangement of soil particles and their aggregation, therefore the structural variation in soils was useful to differentiate the horizons. The structure of the MGT, NHA, BHI, KGI, GTT and KNH soil series were found to be subangular blocky throughout the soil profile, whereas in DSI, KMP, RNL and MAN series varied ie., sub angular blocky at the surface and angular blocky in the subsurface horizons. The blocky structure i.e., angular and 
sub-angular blocky were attributed to the presence of higher quantities of clay fraction (Sharma et al. 2004) ${ }^{[8]}$.

The dry consistence of all the soil series is slightly hard in surface and subsurface horizons. Moist consistence of surface and subsurface horizons are friable in all the soil series. Slightly sticky and slightly plastic consistence was observed in most of the soil series except in DSI, RNL and KMP series where the subsurface horizons found to be very sticky and very plastic and in KMP series it was found to be moderately sticky to slightly plastic. Presence of sticky and plastic to very sticky and very plastic, firm to very firm and slightly hard to very hard consistence in wet, most and dry conditions, respectively may be due to high clay content of soil (Sarkar et al. 2001) and also due to dominance of smectitic clay mineral (Leelavathi et al. 2010) ${ }^{[5,3]}$.

\section{Physical and Chemical Properties of Soils}

The physico - chemical properties of soils are given in Table 3. All the soils are clayey in texture both in surface and subsurface horizons except in NHA, KGI and KNH series and increase in clay content with depth in MAN (69 to 76\%) and DSI (67 to $72 \%$ ) series. This might be due to downward translocation of finer particles from the surface layers (Murthy 1988) ${ }^{[4]}$.
In all the soil series, $\mathrm{pH}$ ranged from neutral to strongly alkaline. The soil $\mathrm{pH}$ was found to be strongly alkaline in RNL (8.42 to 8.63) and MAN (8.39 to 8.49) series both in surface and subsurface horizons. The high $\mathrm{pH}$ in black soils was due to calcareous nature and the accumulation of bases in the solum as they were poorly leached. This wide variation was attributed to the nature of the parent material, leaching, presence of calcium carbonate and exchangeable sodium (Shalima Devi and Anil Kumar 2010) ${ }^{[7]}$.

The organic carbon content was high $(>0.75 \%)$ in surface and subsurface horizons of all the soil series. The $\mathrm{CaCO}_{3}$ content was higher in RNL (8.22 to $9.3 \%$ ) and MAN (5.7 to $8.7 \%$ ) series and the higher $\mathrm{CaCO} 3$ content might be due to semiarid climate which is responsible for the pedogenic processes resulting in the depletion of $\mathrm{Ca}^{+2}$ ions from the soil solution in the form of calcretes (Ashok Kumar and Jagdish Prasad 2010) [1].

The CEC was found to be high in DSI [58.46 to $62.40 \mathrm{cmol}$ $\left.(\mathrm{p}+) \mathrm{kg}^{1}{ }^{1}\right]$ and RNL [60.0 to $\left.63 \mathrm{cmol}(\mathrm{p}+) \mathrm{kg}^{-1}\right]$ series. The ratio between CEC and clay ranged from 0.41 to 2.04 and the CEC: Clay ratio was used to identify the clay mineralogy (Ashok Kumar and Jagdish Prasad 2010) ${ }^{[1]}$.

Table 3: Physico- chemical properties of soils of the parts of Alur, Khairat, Matki and Padasavli subwatersheds

\begin{tabular}{|c|c|c|c|c|c|c|c|c|c|c|c|c|c|c|c|c|}
\hline \multirow{2}{*}{ Horizon } & \multirow{2}{*}{$\begin{array}{c}\text { Depth } \\
\text { (cm) }\end{array}$} & \multicolumn{3}{|c|}{ Particle- size distribution (\%) } & \multirow{2}{*}{$\underset{(1: 2.5)}{\text { pH }}$} & \multirow{2}{*}{$\begin{array}{c}\text { EC } \\
\left(\mathbf{d S m}^{-1}\right)\end{array}$} & \multirow{2}{*}{$\begin{array}{l}\text { OC } \\
(\%)\end{array}$} & \multirow{2}{*}{$\begin{array}{c}\mathrm{CaCO}_{3} \\
(\%)\end{array}$} & \multicolumn{4}{|c|}{$\begin{array}{c}\text { Exchangeable bases } \\
\left(\mathbf{c m o l}(\mathbf{p}+) \mathbf{k g}^{-1)}\right.\end{array}$} & \multirow{2}{*}{$\begin{array}{c}\text { CEC } \\
\left(\mathrm{cmol}^{(p+)}\right. \\
\left.\mathrm{kg}^{1}{ }^{1}\right)\end{array}$} & \multirow{2}{*}{$\begin{array}{c}\text { CEC/ } \\
\text { Clay ratio }\end{array}$} & \multirow{2}{*}{$\begin{array}{l}\text { BS } \\
(\%)\end{array}$} & \multirow{2}{*}{$\begin{array}{l}\text { ESP } \\
(\%)\end{array}$} \\
\hline & & $\begin{array}{c}\text { Sand } \\
(2.00 .05)\end{array}$ & $\left|\begin{array}{c}\text { Silt }(0.05 \\
0.002)\end{array}\right|$ & \begin{tabular}{|c|} 
Clay \\
$(<0.002)$
\end{tabular} & & & & & $\mathbf{C a}$ & Mg & $\mathbf{K}$ & $\mathbf{N a}$ & & & & \\
\hline \multicolumn{17}{|c|}{ Soils of Basalt landscape } \\
\hline \multicolumn{17}{|c|}{ Margutti (MGT) series } \\
\hline Ap & $0-15$ & 10.68 & 31.06 & 58.26 & 6.83 & 0.27 & 0.85 & 3.72 & - & - & 0.66 & 0.09 & 46.27 & 0.79 & 100 & 0.18 \\
\hline $\mathrm{AC}$ & $15-25$ & 38.07 & 20.72 & 41.21 & 7.41 & 0.11 & 0.42 & 3.42 & - & - & 0.27 & 0.24 & 46.37 & 1.13 & 100 & 0.52 \\
\hline \multicolumn{2}{|c|}{ Mean } & 24.38 & 25.89 & 49.73 & 7.12 & 0.19 & 0.64 & 3.57 & - & - & 0.46 & 0.16 & & 0.96 & 100.00 & 0.35 \\
\hline \multicolumn{17}{|c|}{ Novinihala (NHA) series } \\
\hline Ap & $0-15$ & 52.62 & 26.66 & 20.72 & 7.09 & 0.11 & 0.70 & 0.00 & 18.96 & 9.75 & 0.08 & 0.06 & 30.55 & 1.47 & 94 & 0.20 \\
\hline $\mathrm{Bw}$ & $15-40$ & 41.84 & 21.29 & 36.87 & 7.79 & 0.05 & 0.66 & 0.00 & 27.56 & 14.20 & 0.09 & 0.32 & 48.11 & 1.30 & 88 & 0.67 \\
\hline \multicolumn{2}{|c|}{ Mean } & 47.23 & 23.98 & 28.79 & 7.44 & 0.08 & 0.68 & 0.00 & 23.26 & 11.98 & 0.09 & 0.19 & & 1.39 & 91.06 & 0.44 \\
\hline \multicolumn{17}{|c|}{ Bhimanahalli (BHI) series } \\
\hline $\mathrm{Ap}$ & $0-6$ & 41.40 & 17.80 & 40.81 & 6.81 & 0.10 & 0.73 & 2.46 & - & - & 0.15 & 0.05 & 28.03 & 0.69 & 100 & 0.18 \\
\hline Bw1 & $6-25$ & 44 & 82 & 50.74 & 7.37 & 0.07 & 0.77 & 2.70 & - & - & 0.05 & 0.10 & & 58 & 100 & 0.29 \\
\hline $\mathrm{CB}$ & $25-45$ & .17 & .12 & 14.71 & 7.70 & 0.06 & 0.54 & 3.66 & - & - & 0.02 & 0.51 & & 2.04 & 100 & 1.71 \\
\hline \multicolumn{2}{|c|}{ Mean } & 51.67 & 12.91 & 35.42 & 7.29 & 0.08 & 0.68 & 2.94 & - & - & 0.07 & 0.22 & & 1.13 & 100.00 & 0.72 \\
\hline \multicolumn{17}{|c|}{ Kalamundargi (KGI) series } \\
\hline Ap & $0-10$ & 0.04 & 23.67 & 36.29 & 7.15 & 0.12 & 1.05 & 3.90 & - & - & 0.28 & 0.11 & 40.32 & 1.11 & 100 & 0.27 \\
\hline $\mathrm{Bw}$ & $10-28$ & 38.51 & 23.28 & 38.20 & 7.42 & 0.16 & 1.17 & 3.66 & - & - & 0.17 & 0.35 & .81 & 1.57 & 100 & 0.58 \\
\hline \multicolumn{2}{|c|}{ Mean } & 39.28 & 23.47 & 37.25 & 7.29 & 0.14 & 1.11 & 3.78 & & & 0.22 & 0.23 & 50.06 & 1.34 & 100.00 & 0.43 \\
\hline \multicolumn{17}{|c|}{ Dinsi (DSI) series } \\
\hline Ap & $0-10$ & 12.90 & 20.53 & 66.57 & 7.00 & 0.11 & 1.13 & 4.32 & - & - & 0.18 & 0.17 & & & 100 & 0.27 \\
\hline Bw1 & $10-30$ & & 23.91 & 72.84 & 7.24 & 0.08 & 1.13 & 4.86 & - & - & 0.12 & 0.15 & & 0.84 & 100 & 0.25 \\
\hline Bw2 & $30-50$ & & 24.91 & 72.36 & 7.50 & 0.10 & 1.09 & 4.14 & - & - & \begin{tabular}{|l|}
0.14 \\
\end{tabular} & 0.19 & 58.46 & 0.81 & 100 & 0.32 \\
\hline Bw3 & $50-75$ & & 4.35 & 72.28 & 7.56 & 0.10 & 1.13 & 4.08 & - & - & \begin{tabular}{|l|}
0.17 \\
\end{tabular} & 0.17 & 2.40 & 0.86 & 100 & 0.27 \\
\hline $\mathrm{Me}$ & & 5.56 & 23.43 & 71.01 & 7.33 & 0.09 & 1.12 & 4.35 & - & - & 0.15 & 0.17 & 61.03 & 0.86 & 100.00 & \begin{tabular}{|l|}
0.28 \\
\end{tabular} \\
\hline & & & & & & & itti (G & T) ser & & & & & & & & \\
\hline Ap & $0-15$ & 3.40 & 25.23 & 71.37 & 6.46 & 0.08 & \begin{tabular}{|l|}
1.10 \\
\end{tabular} & 0.00 & 23.46 & \begin{tabular}{|l|}
10.89 \\
\end{tabular} & \begin{tabular}{|l|}
0.18 \\
\end{tabular} & 0.22 & 38.19 & 0.54 & 91 & 0.57 \\
\hline Bw1 & $15-40$ & 51 & 18.85 & 77.63 & 6.55 & 0.05 & 1.06 & 0.00 & 18.69 & \begin{tabular}{|l|}
8.35 \\
\end{tabular} & \begin{tabular}{|l|}
0.09 \\
\end{tabular} & 0.21 & 36.14 & 0.47 & 76 & 0.57 \\
\hline $\mathrm{BC}$ & \begin{tabular}{|l}
$40-75$ \\
\end{tabular} & 33.48 & 19.34 & .18 & 6.80 & 0.10 & \begin{tabular}{|l|}
0.90 \\
\end{tabular} & 0.00 & 29.94 & \begin{tabular}{|l|}
12.22 \\
\end{tabular} & \begin{tabular}{|l|}
0.15 \\
\end{tabular} & \begin{tabular}{|l|}
0.27 \\
\end{tabular} & & 0.9 & 100 & 0.64 \\
\hline $\mathrm{Me}$ & & 13.46 & 21.14 & 65.40 & 6.60 & 0.08 & \begin{tabular}{|l|}
1.02 \\
\end{tabular} & 0.00 & 24.03 & 10.49 & \begin{tabular}{|l|}
0.14 \\
\end{tabular} & 0.23 & 34.89 & 39.03 & 0.64 & 0.59 \\
\hline & & & & & & Kamal & lapur & (KMP) s & eries & & & & & & & \\
\hline $\mathrm{Ap}$ & $0-10$ & & 18.80 & 72.90 & 6.69 & \begin{tabular}{l|l}
0.18 \\
\end{tabular} & 0.81 & 3.24 & - & - & \begin{tabular}{|l|}
0.35 \\
\end{tabular} & 0.11 & 43.49 & 0.60 & 100 & 0.25 \\
\hline $\mathrm{AB}$ & $10-30$ & & 19.28 & 72.43 & 7.05 & 0.07 & 0.73 & 3.42 & - & - & \begin{tabular}{|l|}
0.17 \\
\end{tabular} & 0.08 & 40.13 & 0.55 & 100 & 0.20 \\
\hline Bw1 & $30-43$ & 11.70 & 16.58 & 71.72 & 7.15 & 0.10 & 0.54 & 4.32 & - & - & 0.17 & 0.10 & 43.78 & 0.61 & 100 & 0.24 \\
\hline Bw2 & $43-60$ & 11.45 & 21.46 & 67.09 & 7.00 & 0.10 & 0.50 & 2.34 & - & - & \begin{tabular}{|l|}
0.32 \\
\end{tabular} & 0.04 & 44.10 & 0.66 & 100 & 0.08 \\
\hline $\mathrm{BC}$ & 60-85 & 40.34 & 13.27 & 46.39 & 7.80 & 0.09 & 0.38 & 2.85 & - & - & 0.13 & 0.20 & 48.48 & 1.05 & 100 & 0.42 \\
\hline $\mathrm{Me}$ & ean & 16.02 & 17.88 & 66.11 & 7.14 & 0.11 & \begin{tabular}{|l|}
0.59 \\
\end{tabular} & 3.23 & - & - & \begin{tabular}{|l|}
0.23 \\
\end{tabular} & 0.11 & 43.99 & 0.69 & 100 & 0.24 \\
\hline
\end{tabular}




\begin{tabular}{|c|c|c|c|c|c|c|c|c|c|c|c|c|c|c|c|c|}
\hline \multicolumn{17}{|c|}{ Rajanala (RNL) series } \\
\hline Ap & $0-18$ & 10.17 & 28.16 & 61.68 & 8.42 & 0.22 & 0.97 & 9.36 & - & - & 0.50 & 0.13 & 60.00 & 0.97 & 100 & 0.21 \\
\hline A1 & $18-40$ & 9.83 & 24.55 & 65.62 & 8.63 & 0.18 & 0.58 & 8.70 & - & - & 0.37 & 0.12 & 61.82 & 0.94 & 100 & 0.20 \\
\hline Bss1 & $40-60$ & 6.59 & 22.19 & 71.22 & 8.55 & 0.20 & 0.50 & 8.64 & - & - & 0.28 & 0.21 & 63.26 & 0.89 & 100 & 0.32 \\
\hline Bss2 & 60-105 & 6.13 & 16.86 & 77.01 & 8.57 & 0.20 & 0.62 & 9.06 & - & - & 0.27 & 0.18 & 61.44 & 0.80 & 100 & 0.30 \\
\hline Bss3 & $105-150$ & 12.37 & 20.36 & 67.27 & 8.62 & 0.22 & 0.55 & 8.22 & - & - & 0.47 & 0.13 & 60.86 & 0.90 & 100 & 0.22 \\
\hline & ean & 9.02 & 22.42 & 68.56 & 8.56 & 0.20 & 0.64 & 8.80 & & & 0.38 & 0.15 & 61.48 & 0.90 & 100.00 & 0.25 \\
\hline \multicolumn{17}{|c|}{ Mahagaon (MAN) series } \\
\hline Ap & $0-20$ & 3.87 & 26.80 & 69.33 & 8.35 & 0.20 & 0.87 & 5.70 & - & - & 0.53 & 0.08 & 58.46 & 0.84 & 100 & 0.14 \\
\hline A1 & $20-45$ & 6.05 & 24.97 & 68.97 & 8.49 & 0.17 & 0.63 & 6.84 & - & - & 0.25 & 0.10 & 56.54 & 0.82 & 100 & 0.17 \\
\hline Bss1 & $45-75$ & 5.08 & 17.69 & 77.23 & 8.43 & 0.19 & 0.66 & 7.38 & - & - & 0.22 & 0.11 & 58.66 & 0.76 & 100 & 0.18 \\
\hline Bss2 & $75-110$ & 4.74 & 24.52 & 70.74 & 8.46 & 0.23 & 0.58 & 6.00 & - & - & 0.30 & 0.13 & 59.04 & 0.83 & 100 & 0.22 \\
\hline Bss3 & $110-140$ & 5.44 & 18.28 & 76.28 & 8.43 & 0.22 & 0.43 & 7.50 & - & - & 0.27 & 0.18 & 57.12 & 0.75 & 100 & 0.31 \\
\hline & \begin{tabular}{|l|l|} 
ean & \\
\end{tabular} & 4.20 & 22.45 & 72.51 & 8.6 & 0.24 & 0.46 & 8.70 & & & 0.28 & 0.23 & 39.36 & 0.80 & 100 & 0.58 \\
\hline \multicolumn{17}{|c|}{ Soils of Laterite landscape } \\
\hline \multicolumn{17}{|c|}{ Kinhi (KNH) series } \\
\hline Ap & $0-18$ & 44.63 & 18.45 & 36.92 & 6.35 & 0.07 & 0.73 & 1.26 & - & - & 0.32 & 0.02 & 15.26 & 0.41 & 100 & 0.16 \\
\hline
\end{tabular}

\section{Classification of Soils}

The 10-soil series under study are classified based on morphological, physical and chemical properties according to Soil Taxonomy (Soil Survey Staff, 2014) ${ }^{[9]}$. At higher categories (Order) the presence or absence of diagnostic horizons which are indications of pedogenic process were considered. At Sub-order level, the moisture and temperature regimes were used. At lower categories (Great group, Subgroup and Family), diagnostic subsurface horizons, soil depth, mineralogy, texture, soil chemical properties and drainage are considered. The classification of the soil series under study is given in Table 4.

In this study, three soil orders were identified namely, Entisols, Inceptisols and Vertisols. Among the ten-soil series Margutti (MGT) and Kinhi (KNH) classified as "Entisols", because there was no evidence of development of pedogenic horizons. So it was classified as Orthents at great group level and as Ustorthents due to the prevailing soil moisture regime as "Ustic".

Novinihala (NHA), Bhimanahalli (BHI), Kalamundargi (KGI) and Gutti (GTT) series were classified as "Inceptisols" owing to the presence of cambic horizon and as the moisture regime is Ustic classified as Ustepts at suborder level.

Dinsi (DSI), Kamalapur (KMP), Rajanala (RNL) and Mahagaon (MAN) soil series were classified as "Vertisols", at order level as these pedons did not have lithic or paralithic contact within $50 \mathrm{~cm}$ of soil surface and had a weighted average of more than 30 per cent clay in all the horizons down to a depth of $1 \mathrm{~m}$ and had cracks that open and close periodically. These series have a layer of more than $25 \mathrm{~cm}$ thick, with an upper boundary within $100 \mathrm{~cm}$ of mineral soil surface that had intersecting slickensides. These series key out as Vertisols and Usterts at suborder level as they have cracks in normal year that are $5 \mathrm{~mm}$ or more through a thickness of $25 \mathrm{~cm}$ or more within $50 \mathrm{~cm}$ of mineral soil surface for 90 or more cumulative days per year. They further key out as Haplusterts at great group level as they do not have salic, gypsic or calcic horizons and at subgroup level classified as Typic Haplusterts as it did not qualify for any other subgroup under Haplusterts.

Table 4: Classification of soils of Parts of Alur, Khairat, Matki and Padasavli Subwatersheds

\begin{tabular}{|c|c|c|c|c|c|c|}
\hline Sl. No & Order & Suborder & Great group & Sub group & Family & Soil series \\
\hline 1 & Entisols & Orthents & Ustorthents & (Paralithic) Ustorthents & $\begin{array}{c}\text { Clayey, mixed, isohyperthemic, (Paralithic) } \\
\text { Ustorthents }\end{array}$ & Margutti (MGT) \\
\hline 2 & Inceptisols & Ustepts & Haplustepts & Paralithic) Haplustepts & $\begin{array}{c}\text { Clayey, mixed, isohyperthemic, (Paralithic) } \\
\text { Haplustepts }\end{array}$ & $\begin{array}{c}\text { Novinihala (NHA) } \\
\text { Bhimanahalli (BHI) }\end{array}$ \\
\hline 3 & Inceptisols & Ustepts & Haplustepts & Lithic Haplustepts & $\begin{array}{c}\text { Loamy- skeletal, mixed isohyperthermic, } \\
\text { Lithic Haplustepts }\end{array}$ & $\begin{array}{c}\text { Kalamundargi } \\
\text { (KGI) }\end{array}$ \\
\hline 4 & Inceptisols & Ustepts & Haplustepts & Typic Haplustepts & $\begin{array}{c}\text { Fine, montmorillonite, isohyperthermic, } \\
\text { Typic Haplustepts }\end{array}$ & Gutti (GTT) \\
\hline 5 & Entisols & Orthents & Ustorthents & (Paralithic) Ustorthents & $\begin{array}{c}\text { Clayey-skeletal, mixed, isohyperthemic, } \\
\text { (Paralithic) Ustorthents }\end{array}$ & Kinhi (KNH) \\
\hline 6 & Vertisols & Usterts & Haplusterts & Typic Haplusterts & $\begin{array}{c}\text { Very fine, smectitic, isohyperthermic } \\
\text { (calcareous), Typic Haplusterts }\end{array}$ & $\begin{array}{c}\text { Rajanala (RNL) } \\
\text { Mahagaon (MAN) }\end{array}$ \\
\hline 7 & Vertisols & Usterts & Haplusterts & Typic Haplusterts & $\begin{array}{c}\text { Very fine, smectitic, isohyperthermic, } \\
\text { Typic Haplusterts }\end{array}$ & $\begin{array}{c}\text { Dinsi (DSI) } \\
\text { Kamalapur (KMP) }\end{array}$ \\
\hline
\end{tabular}

\section{Acknowledgements}

Authors acknowledge Watershed Development Department, Government of Karnataka and World Bank for providing financial support to carry out the study under Sujala-III project. The technical support provided by Sujala staff is greatly acknowledged.

\section{References}

1 Ashok Kumar HP, Jagdish Prasad. Some typical sugarcane growing soils of Ahmednagar district of Maharashtra. Their characterization, classification and nutritional status of soils and plants. Journal of the Indian Society of Soil Science. 2010; 58:257-266.

2 Geetha Sireesha PV, Naidu MVS. Studies on genesis, characterization and classification of soils in semi-arid agro-ecological region: A case study in Banaganapalle 
Mandal of Kurnool district, Andhra Pradesh. Journal of the Indian Society of Soil Science. 2013; 61:167-178.

3 Leelavathi GP, Naidu MVS, Ramavatharam N, Karuna Sagar G. Clay mineralogy of soils formed on granitegneiss of Chittoor district, Andhra Pradesh. Journal of the Indian Society of Soil Science. 2010; 58:376-383.

4 Murthy ASP. Distribution, properties and management of Vertisols in India. Advances in Soil Science. 1988; 8:153-214

5 Sarkar D, Gangopadhyay SK, Velayutham M. Soil toposequence relationship and classification in lower outlier of Chhotanagpur plateau. Agropedology. 2001; 11:29-36.

6 Sarma VAK, Krishnan P, Budihal SL. Laboratory Manual, Tech. Bull. 23, NBSS \& LUP, Nagpur, 1987.

7 Shalima Devi GM, Anil Kumar KS. Characterization and classification of coffee growing soils of Karnataka. Journal of the Indian Society of Soil Science. 2010; 58:125-131.

8 Sharma SS, Totawat KL, Shyampura RL. Characterization and classification of salt-affected soils of southern Rajasthan. Journal of the Indian Society of Soil Science. 2004; 52:209-213.

9 Soil Survey Staff. Keys to Soil Taxonomy, 12th edition, USDA, Natural Resource Conservation Service, Washington, DC, 2014.

10 Tripathi D, Verma JR, Patial KS, Karan Singh. Characteristics, classification and suitability of soils for major crops of Kiar-Nagali micro-watershed in NorthWest Himalayas. Journal of the Indian Society of Soil Science. 2006; 54:131-136.

11 Walia CS, Rao YS. Characteristics and classification of some soils of Trans-Yamuna plains. Journal of the Indian Society of Soil Science. 1997; 45:156-162. 